\title{
ARTICLE \\ hvTRA, a novel TRAIL receptor agonist, induces apoptosis and sustained growth retardation in melanoma
}

\author{
Karianne G Fleten ${ }^{1}$, Vivi Ann Flørenes ${ }^{2}$, Lina Prasmickaite ${ }^{1}$, Oliver Hill ${ }^{3}$, Jaromir Sykora ${ }^{3}$, Gunhild M Mælandsmo ${ }^{1,4}$ \\ and Birgit Engesæter ${ }^{1}$
}

In recent years, new treatment options for malignant melanoma patients have enhanced the overall survival for selected patients. Despite new hope, most melanoma patients still relapse with drug-resistant tumors or experience intrinsic resistance to the therapy. Therefore, novel treatment modalities beneficial for subgroups of patients are needed. TRAIL receptor agonists have been suggested as promising candidates for use in cancer treatment as they preferentially induce apoptosis in cancer cells. Unfortunately, the first generation of TRAIL receptor agonists showed poor clinical efficacy. hvTRA is a second-generation TRAIL receptor agonist with improved composition giving increased potency, and in the present study, we showed hvTRA-induced activation of apoptosis leading to an efficient and sustained reduction in melanoma cell growth in cell lines and xenograft models. Furthermore, the potential of hvTRA in a clinical setting was demonstrated by showing efficacy on tumor cells harvested from melanoma patients with lymph node metastasis in an ex vivo drug sensitivity assay. Inhibition of mutated BRAF has been shown to regulate proteins in the intrinsic apoptotic pathway, making the cells more susceptible for apoptosis induction. In an attempt to increase the efficacy of hvTRA, combination treatment with the mutated BRAF inhibitor vemurafenib was investigated. A synergistic effect by the combination was observed for several cell lines in vitro, and an initial cytotoxic effect was observed in vivo. Unfortunately, the initial increased reduction in tumor growth compared with hvTRA mono treatment was not sustained, and this was related to downregulation of the DR5 level by vemurafenib. Altogether, the presented data imply that hvTRA efficiently induce apoptosis and growth delay in melanoma models and patient material, and the potential of this TRAIL receptor agonist should be further evaluated for treatment of subgroups of melanoma patients.

Cell Death Discovery (2016) 2, 16081; doi:10.1038/cddiscovery.2016.81; published online 12 December 2016

\section{INTRODUCTION}

Malignant melanoma is a highly metastatic disease with poor survival rate. Despite recent advancements leading to novel treatment options, such as the mutated BRAF inhibitor, vemurafenib and the immune activator, ipilimumab, there is still no curative treatment for the majority of patients with advanced disease. $^{1,2}$ New therapeutic options are therefore of great importance in order to improve clinical outcomes. TRAIL receptor agonists (TRAs) have been suggested as promising anticancer candidates as they preferentially induce apoptosis in tumor cells, while normal cells are generally unaffected. ${ }^{3,4}$ TRAs induce apoptosis by binding to Death Receptor 4 (DR4/TRAIL receptor-1) or Death Receptor 5 (DR5/TRAIL receptor-2), leading to receptor clustering and activation of the extrinsic apoptotic pathway. ${ }^{5}$ In contrast to the promising results obtained in preclinical models, all clinical trials trying to establish TRAs as drugs for human use have failed so far. ${ }^{6-13}$ The reasons for the observed clinical failures of the first-generation TRAs are related to short in vivo exposure of the drug due to its fast elimination, ${ }^{11}$ insufficient multimerization efficacy in vivo, ${ }^{14}$ low expression of the TRAIL-receptors DR4 or DR5, ${ }^{15,16}$ loss or incomplete activation of pro-caspases 3 and $8^{15,17,18}$ and upregulation of the anti-apoptotic protein Bcl-xL. ${ }^{19}$ Despite the negative clinical outcomes, there are still activities ongoing to develop TRAs for clinical use due to the potential benefits for patients. One second-generation development is represented by the recently described synthetic fusion protein APG350 (hvTRA). ${ }^{20}$ It consists of two trivalent single-chain TRAIL receptor-binding domains, which are covalently linked to each other by a silenced IgG1-Fc domain, resulting in a hexavalent TRAIL receptor agonist (hvTRA). Due to its unique molecular layout, hvTRA facilitates close-proximity multimerization of DR4/ DR5 and thereby induces efficient activation of intratumoral apoptosis that is independent of Fcy receptor-driven secondary crosslinking events in vivo. Consequently, hvTRA has been proven to be more efficient than other TRAs in preclinical models of various cancer types. ${ }^{20}$

Combining therapies, through cooperative inhibition or stimulation of multiple targets, offer a promising approach for effective treatment, and different drugs have been combined with TRAs in attempts to increase the efficacy of the drugs. ${ }^{21-25}$ Approximately $50 \%$ of all melanoma patients harbor mutated BRAF causing constitutive active MAPK/ERK pathway. Inhibition of the MAPK/ ERK pathway can influence survival by affecting proteins in the intrinsic apoptotic pathway such as Bim, BMF and Bad, ${ }^{26-29}$ and reduce the stability of the anti-apoptotic protein $\mathrm{Mcl}-1,{ }^{27}$ thus priming the cells for apoptosis induction. ${ }^{30,31}$

\footnotetext{
${ }^{1}$ Department of Tumor Biology, Oslo University Hospital, The Norwegian Radium Hospital, Oslo, Norway; ${ }^{2}$ Department of Pathology, Oslo University Hospital, The Norwegian Radium Hospital, Oslo, Norway; ${ }^{3}$ Apogenix GmbH, Im Neuenheimer Feld, Heidelberg, Germany and ${ }^{4}$ Department of Pharmacy, Faculty of Health Sciences, University of Troms $\varnothing$, Tromsø, Norway.

Correspondence: KG Fleten (kaflet@rr-research.no)

Received 14 April 2016; revised 13 September 2016; accepted 23 September 2016; Edited by I Harris
} 
MAPK/ERK pathway inhibition together with TRAs is a potentially beneficial combination that stimulates both the extrinsic and intrinsic apoptotic pathways. However, conflicting in vitro results for the combination have been reported. ${ }^{21,32}$

The aims of this study were to investigate the efficacy of hvTRA alone and in combination with the mutated BRAF inhibitor vemurafenib in melanoma cell lines, xenograft model and patient materials. Our results show that hvTRA effectively reduce the viability of melanoma cells both in vitro and in vivo and strongly encourage further evaluation of hvTRA alone. However, vemurafenib-induced downregulation of DR5 seems to represent a limiting factor for therapeutic success of combining hvTRA and vemurafenib.

\section{RESULTS}

hvTRA reduce melanoma cell viability, lung tissue colonization and tumor growth

The potential of hvTRA to reduce cell viability was examined in seven melanoma cell lines. As shown in Figure 1a, all cell lines demonstrated a dose-dependent reduction in viability after treatment with hvTRA for $72 \mathrm{~h}$. The strongest response was observed in Patient-3-pre, Patient-3-post and WM1366, whereas A375 and Melmet 5 showed the least responsiveness. Cleavage of pro-caspases 3 and 8, Bid and PARP indicate that hvTRA induce apoptosis through the extrinsic apoptotic pathway (Supplementary Figure 1).

As melanomas often metastasize to the lungs, colonization of lung tissue slices in the ex vivo PUMA assay ${ }^{33}$ was used to further explore the efficacy of hvTRA. hvTRA significantly reduced the growth of Melmet 5-GFP-Luc cells measured by decreased GFP fluorescent intensity compared with the intensity in untreated lung slices on days 9 and 14 (Figure 1b). The efficacy of hvTRA was also evaluated in vivo, where Melmet 5 xenografts were treated with two different concentrations of hvTRA $(0.3$ or $3 \mathrm{mg} / \mathrm{kg})$ for 5 days. Similar to the in vitro experiments, a dose-dependent reduction in tumor volume was observed (Figure 1c). In summary, the results suggest that hvTRA inhibits melanoma cell viability in various models in vitro, ex vivo and in vivo.

hvTRA and vemurafenib induce synergistic effects in vitro

Previous studies have shown increased efficacy of TRAs when combined with inhibitors of mutated BRAF. ${ }^{21,34}$ In this study, the combination hvTRA and the clinically used BRAF inhibitor, vemurafenib, was examined. The effect of vemurafenib alone was established in selected cell lines. A strong dose-dependent response was observed in A375, Melmet 5 and Patient-3-pre, whereas in LOX, Melmet 1, Patient-3-post and the wild-type BRAF cell line WM1366 the response was modest (Supplementary Figure 2). Subsequently, we exposed the cells to hvTRA ( 0.3 and $1 \mu \mathrm{g} / \mathrm{ml}$ ) in combination with 0.3 (data not shown) and $1 \mu \mathrm{M}$ vemurafenib (Figure 2a). Potential synergy was evaluated by calculating the combination index $(\mathrm{Cl})$ using the Calcusyn software (Supplementary Table 1). The combination had a synergistic effect in A375, Melmet 5, LOX, Patient-3-pre and Patient-3-post cells, whereas an antagonistic effect was observed in Melmet 1.

Compared with monolayer cultures, spheroids are supposed to better reflect the in vivo conditions with respect to drug efficacy. ${ }^{35,36}$ When Melmet 1 spheroids were treated with hvTRA and vemurafenib alone and in combination, we found, in contrast to the monolayer cultures, that the drug combination was more effective than hvTRA $(P=0.091)$ and vemurafenib $(P=0.045)$ alone (Figure $2 \mathrm{~b}$ ). Likewise, Melmet 5 spheroids were significantly more responsive to the combined treatment than to hvTRA $(P<0.001)$ and vemurafenib $(P=0.028)$ alone.
Combining hvTRA and vemurafenib gives an increased initial antitumor effect in vivo

Altogether, our in vitro results suggest that combining hvTRA with vemurafenib could be effective at least in a subset of melanomas. To evaluate the efficacy in vivo, the growth of Melmet 5 xenografts were followed while treating the mice with hvTRA $(3 \mathrm{mg} / \mathrm{kg})$, vemurafenib $(50 \mathrm{mg} / \mathrm{kg})$ or the combination as indicated in Figure 3. Both mono treatments inhibited the growth of Melmet 5 xenografts. Xenografts treated with vemurafenib remained stable during the treatment period, while, notably, a regression in size was observed in xenografts treated with hvTRA after day 10. Xenografts treated with both hvTRA and vemurafenib experienced a strong initial decrease in tumor size, not observed for any of the mono treatments, and the tumor volumes were significantly reduced compared with the tumors exposed to any of the mono treatments or controls from days 2 to 11 (insert Figure 3; $P<0.005)$. The tumor volume in the vemurafenib and combination groups increased after seponation, while interestingly, the tumors treated with hvTRA did not start growing again until 8 days after seponation (day 25).

\section{Regulation of DR5 expression by hvTRA and vemurafenib}

Both DR4 and DR5 are expressed in Melmet 5 xenografts (Figure 4), but DR4 may not be functional as response to a DR4 agonist in vitro is lacking (data not shown). DR4 expression in the xenografts seems not to be affected by any of the treatment regimens, whereas DR5 levels were upregulated after 1 and 4 days of hvTRA treatment and strongly downregulated in xenografts treated with vemurafenib or the combination (Figures $4 a$ and $b$ ). Downregulation of DR5 by vemurafenib was also observed in vitro, but only using higher concentrations than utilized in the combination experiments (data not shown). The transcription factor $\mathrm{CHOP}$ and phosphorylation of the transcription factor c-jun positively regulates the transcription of the DR5 gene. ${ }^{37}$ Our data showed increased phosphorylation of c-jun in tumors receiving only hvTRA both on days 1 and 4, corresponding to the observed upregulated DR5 level in the same samples (Figures $4 a$ and b). However, p-c-jun was dephosphorylated in response to vemurafenib treatment. Expression of CHOP was exclusively observed in samples treated with hvTRA, both alone and in combination on day 1. The level of CHOP and phosphorylation of c-jun can be influenced by MAPK/ERK pathway signaling, ${ }^{37}$ and the presented data correspond to the activity of the signaling pathway. Reduced levels of pERK1/2 were observed in response to vemurafenib, indicating reduced MAPK/ERK activity. ${ }^{38}$ Interestingly, hvTRA increased pERK $1 / 2$ levels on day 4 and also slightly counteracted the downregulation of $\mathrm{pERK} 1 / 2$ induced by the BRAF inhibitor on days 1 and 4 (Figures $4 a$ and $b$ ).

\section{Regulation of apoptosis-related proteins by hvTRA and} vemurafenib

As demonstrated for monolayer cultures, hvTRA initiated the extrinsic caspase cascade also in melanoma spheroids (Figure 5a) and xenografts (Figure $5 \mathrm{~b}$ ). This was shown by cleavage of procaspase 8 and Bid. Notably, no differences in the protein levels were observed between spheroids treated only with hvTRA and the combination. However, xenografts harvested after hvTRA treatment for 4 days demonstrated more cleavage of pro-caspase 8 and Bid compared with xenografts treated with both hvTRA and vemurafenib (Figure 5b), suggesting reduced intratumoral apoptosis in the latter group. Cleavage of PARP was only observed clearly in spheroids (Figure 5a), while in xenografts the level of cleaved PARP in hvTRA-treated samples was similar to control (Figure $5 b$ ). The two xenografts treated with combination therapy showed conflicting results as one xenograft showed higher degree of PARP and pro-caspase 3 cleavage compared with xenografts 


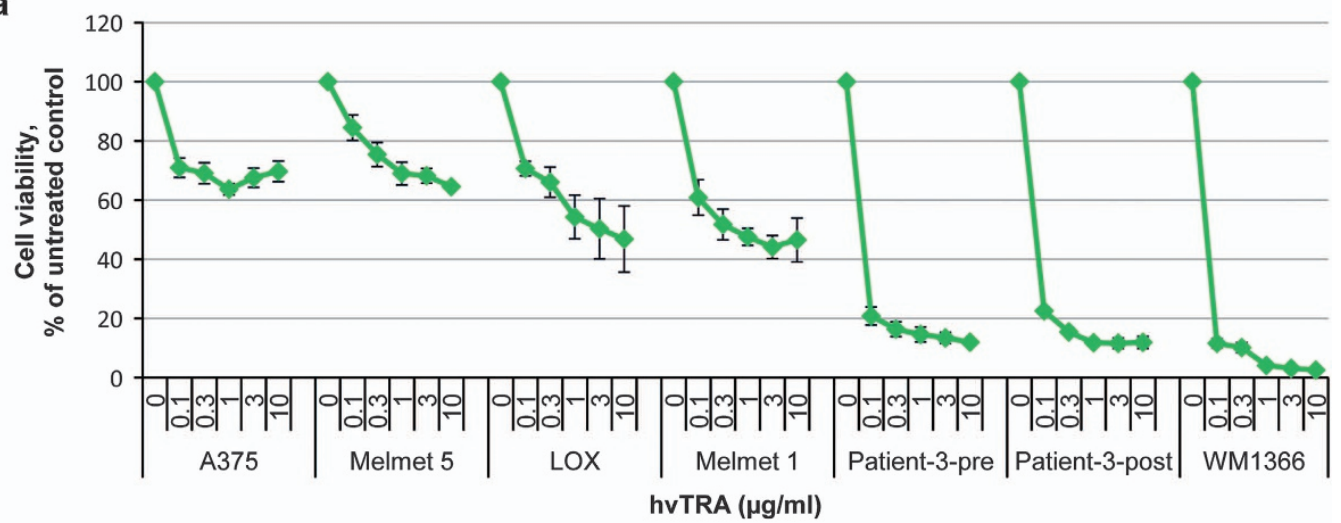

b

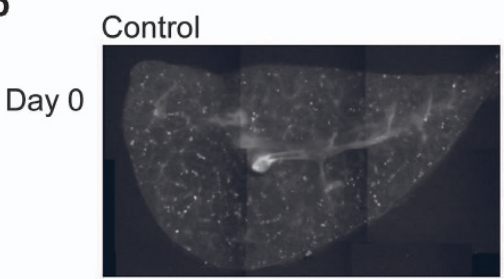

hvTRA

Day 9
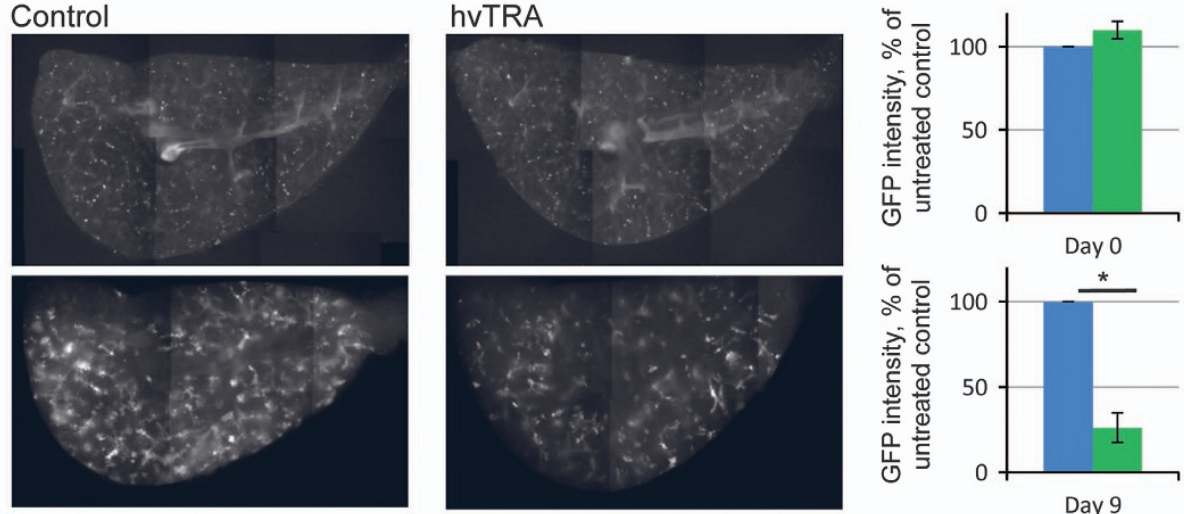

Day 14
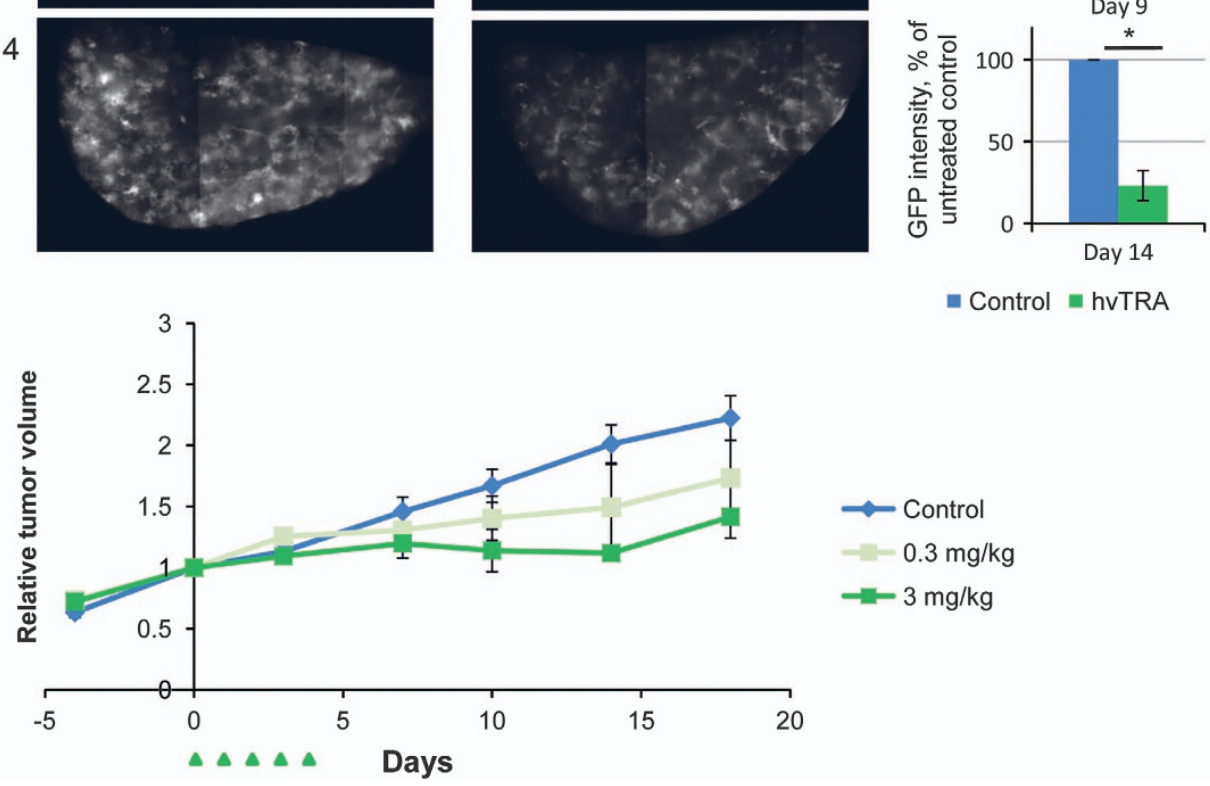

Figure 1. hvTRA reduced cell viability in melanoma cells and initiate the extrinsic apoptotic pathway. (a) Seven melanoma cell lines were exposed to increasing concentrations of hvTRA. Cell viability was measured after $72 \mathrm{~h}$ by the MTS assay. The experiments were repeated three times and error bars represent \pm S.E.M. (b) Left panel: representative pictures of the pulmonary metastasis assay (PuMA) showing growth of Melmet 5-GFP cells ex vivo in lung tissue exposed to hvTRA $(0.25 \mu \mathrm{g} / \mathrm{ml})$ at day 0,9 and 14 . Right panel: quantification of fluorescence from the Melmet 5-GFP cells in the lung tissue section using ImageJ. The fluorescent intensity of the control samples was set to $100 \%$ for each time point, and the intensity of hvTRA-treated samples were related to the control from the same day. Four biological replicates were performed with three to four technical replicates. (c) Melmet 5 xenografts grown subcutaneously in nude mice were treated with 0.3 or $3 \mathrm{mg} / \mathrm{kg}$ hvTRA (i.v. injection) as indicated in the treatment schedule depicted in the figure by 4 . The tumors were measured twice a week using a caliper, and are presented as relative tumor volume related to the volume of the tumor at the initiation of the treatment. Three mice (six tumors) were included in the control group, and two mice (four tumors) were included in both groups treated with hvTRA. Error bars represent \pm S.E.M. ${ }^{*} P<0.05$. 
a

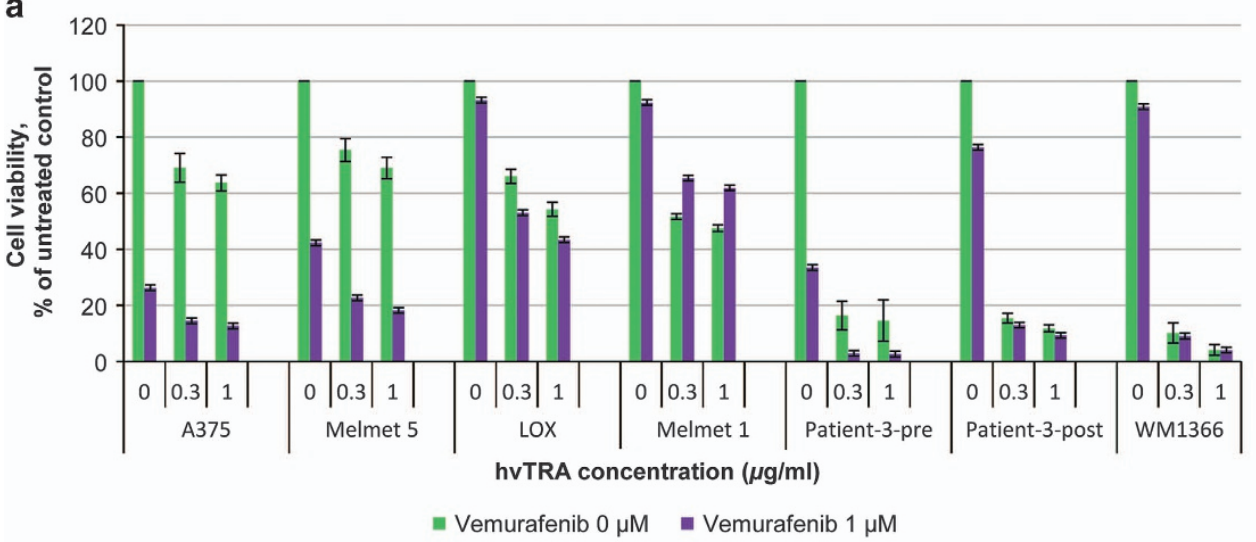

b

Melmet 1 spheroids

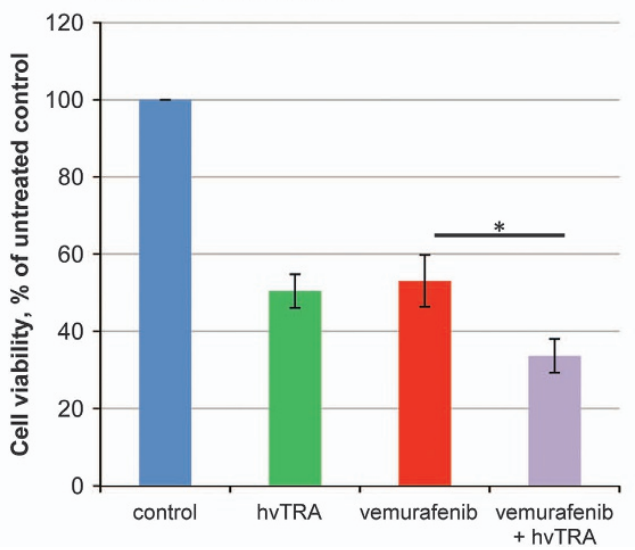

Melmet 5 spheroids

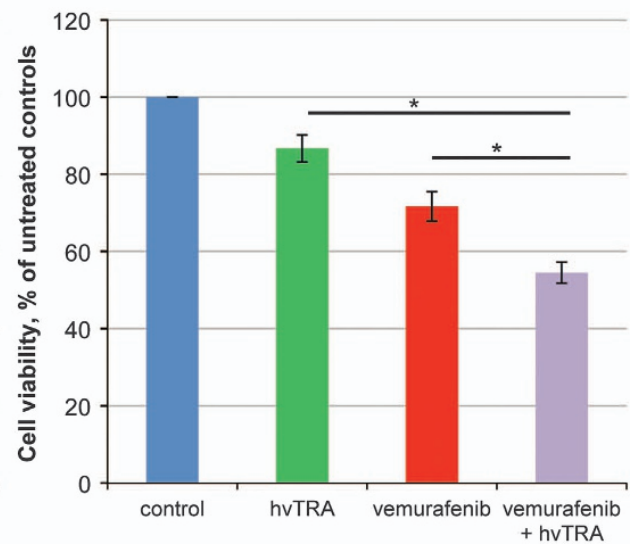

Figure 2. In vitro response to hvTRA, vemurafenib and the combination. (a) Seven melanoma cell lines were exposed to the combination of vemurafenib $(1 \mu \mathrm{M})$ and hvTRA at selected concentrations (purple bars). The data representing hvTRA mono treatments (green bars) are the same results as shown in Figure $1 \mathrm{a}$. Cell viability was measured after $72 \mathrm{~h}$ by MTS assay. The experiments were repeated three times. (b) Melmet 1 and Melmet 5 were grown as spheroids and treated with hvTRA $(0.5 \mu \mathrm{g} / \mathrm{ml})$, vemurafenib $(0.3 \mu \mathrm{M})$ or the combination. Cell viability was measured using CellTiter-Glo Luminescent assay and measured $96 \mathrm{~h}$ after start of treatment. At least four biological replicated were performed, with four technical replicates. The error bars represent \pm S.E.M. ${ }^{*} P<0.05$.

treated with hvTRA only, while the other showed comparable levels. Cleavage of caspase 3 (Figures $5 a$ and b) was not observed in spheroids or xenografts treated with vemurafenib, indicating that apoptosis is not executed in response to vemurafenib. The level of cleaved caspase 3 in the xenografts was also investigated by IHC (Figure $5 \mathrm{c}$ ). The staining intensity of cleaved caspase 3 was highest following hvTRA alone, replicating the results observed by western immunoblotting, suggesting that vemurafenib inhibits the efficacy of hvTRA.

In an attempt to unravel the molecular effects of the various treatments in more detail, proteins in the intrinsic apoptotic pathway were studied. As shown in Figures $5 \mathrm{~d}$ and e, dephosphorylation of pro-apoptosis Bad ${ }^{\text {ser112 }}$ protein, an indication of activation of the intrinsic apoptotic pathway, was seen following exposure to vemurafenib in both spheroids and xenografts, while Bad was downregulated in response to hvTRA in xenografts. The pro-apoptotic protein Bim-EL was downregulated in hvTRAtreated and upregulated in vemurafenib-treated xenografts. Following combined treatment, the Bim-EL protein level was further increased compared with vemurafenib alone in vivo (Figure 5e), while in vitro Bim-EL level was reduced after combination therapy compared with vemurafenib alone. The anti-apoptotic protein $\mathrm{Mcl}-1$ was downregulated in Melmet 5 spheroids after the combined treatment, while in Melmet 1 spheroids and Melmet 5 xenografts the Mcl-1 level was decreased both after treatment with vemurafenib alone and in combination. hvTRA increased the Mcl-1 levels in vivo.

Reduced vemurafenib dosing does not give a synergistic longterm effect

Our previous in vivo study revealed that hvTRA significantly reduces tumor growth, and combining hvTRA with vemurafenib gave no additional long-term effect. The initial cytotoxic effect observed in the combination group in Figure 3 suggests a potential advantage of the combination. The transient response may however be explained by vemurafenib-induced repression of the DR5 expression (Figure 4). It was therefore of interest to adjust the vemurafenib treatment regime in an attempt to maximize the cytotoxic response and minimize the effect on the TRAIL receptor. Reducing the vemurafenib dose to one-fourth $(50 \mathrm{mg} / \mathrm{kg}$ once a day every second day) resulted in no tumor growth delay in the vemurafenib-treated group (Figure 6a). Xenografts receiving combination treatment showed initial decrease in tumor size, but not as pronounced as with the higher vemurafenib dose. After the initial decrease, the tumor size remained stable. There was a significant difference in tumor volume between xenografts treated with hvTRA and the combination from day 4 to day 10 $(P<0.05)$. In the vemurafenib and the combination-treated xenografts, the DR5 level was slightly lower compared with the 
control (Figure 6b). p-c-jun followed the same trend as DR5 with the highest level in hvTRA-treated samples, and a weak reduction in vemurafenib-treated samples compared with the control. When the frequency of vemurafenib dosing was reduced, the cleavage of Bid and pro-caspases 3 and 8 was similar between hvTRA alone and the combination (Supplementary Figure $3 \mathrm{~A}$ ), which could indicate that hvTRA was able to bind to DR5 and activate the extrinsic apoptotic pathway. However, the concentration of vemurafenib has been too low to give an additional inhibition of tumor growth compared to hvTRA mono treatment.

A slight reduction in DR5 expression was still observed in response to the reduced dosing frequency of vemurafenib (Figure 6b). Therefore, another in vivo experiment was initiated with the intention that DR5 expression would be completely restored between each time vemurafenib was given. Vemurafenib

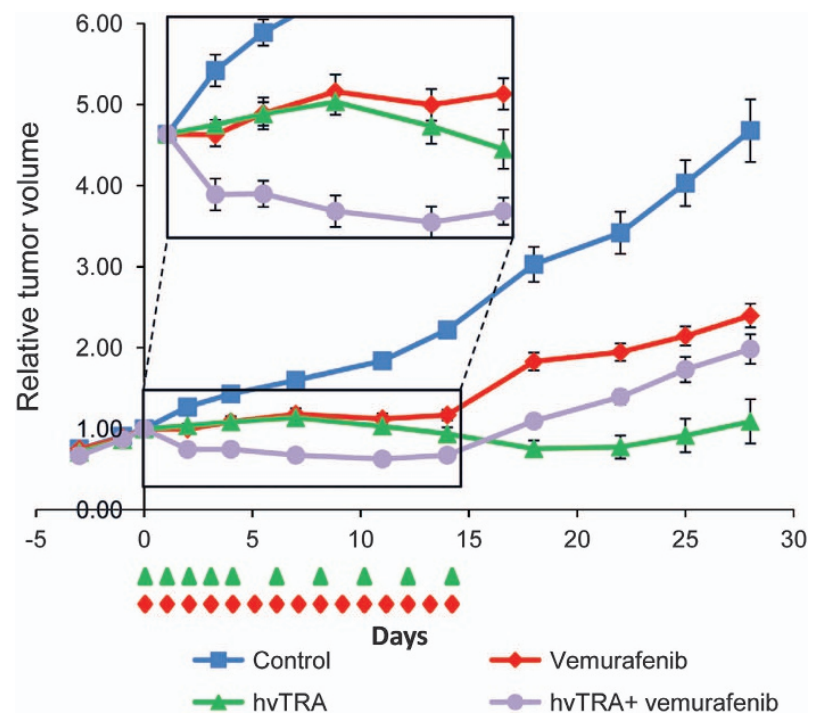

Figure 3. The antitumor effect of hvTRA, vemurafenib and the combination in vivo. Melmet 5 xenografts were treated with hvTRA ( ) $(3 \mathrm{mg} / \mathrm{kg}$, i.v. injection) or vemurafenib ( ) $50 \mathrm{mg} / \mathrm{kg}$, orally twice a day) or the combination of hvTRA and vemurafenib ( $)$ ). The treatment schedule is indicated in the figure by $\Delta$ and $\downarrow$. The tumors were measured twice or thrice a week using a caliper, and are presented as relative tumor volume related to the volume of the tumor at the initiation of the treatment. Eight tumors are included in each group. Error bars represent \pm S.E.M.

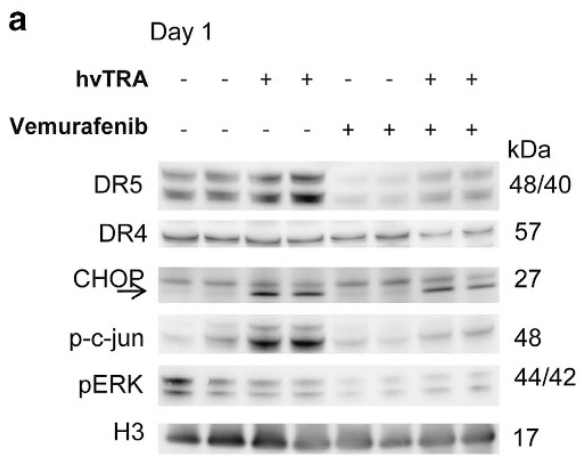

(50 mg/kg) was given twice a day every 8th days, while hvTRA dosing was kept as before (Figure 6c). hvTRA showed similar effect as previously with regression in tumor size after day 10. As observed in the preceding experiments, there was an initial decrease in tumor volume in response to the combination. The subsequent vemurafenib treatment on day 8 induced a slight decrease in tumor volume, while later administrations on days 16 and 24 gave no additional effect. The size of the tumors treated with hvTRA or the combination were reduced to a similar level. DR5 levels were investigated 8 days after the last treatment. No regulation of DR5 were observed in response to vemurafenib, indicating that 8 days are enough for the DR5 levels to return to normal (Figure $6 \mathrm{~d}$ ). In conclusion, our results indicate that combining hvTRA and vemurafenib did not enhance the effect of hvTRA, while hvTRA as mono treatment induced a stable and sustained growth delay in all experiments performed.

hvTRA can reduce cell viability in patient biopsies

To investigate the clinical relevance of hvTRA alone or in combination with vemurafenib, biopsies from lymph node metastases from 11 melanoma patients were collected, cultured as spheroids in vitro and treated with hvTRA $(5 \mu \mathrm{g} / \mathrm{ml})$, vemurafenib $(5 \mu \mathrm{M})$ or the combination (Figure 7$)$. The high concentrations were chosen to ensure detection of response if there was any. hvTRA reduced cell survival in six out of eleven patient biopsies (27-74\% reduction), and increased the viability in two. DR5 levels were investigated by IHC, and the results showed that DR5 expression was required, but did not guarantee a response (Figure 7). Vemurafenib reduced the viability in seven of the eleven investigated patient biopsies, four with and three without the $\mathrm{V} 600 \mathrm{E} / \mathrm{K}$ mutation. Five patient biopsies exposed to combination therapy showed a tendency to decreased cell viability compared with the mono treatments, although it was only significant for patient number 317 . In the remaining biopsies, cell survival after combination treatment was similar or higher than either mono treatments (Figure 7). These results suggest that hvTRA alone or in combination could be beneficial for a subgroup of patients; however, biomarkers are desirable to be able to predict which patients will respond.

\section{DISCUSSION}

It is well accepted that TRAIL induces apoptosis more efficiently in cancer cells than normal cells, ${ }^{3,4}$ and as a consequence different TRAs have been developed. hvTRA is a novel TRA, which has previously shown efficacy in different cancer cell lines in vitro and

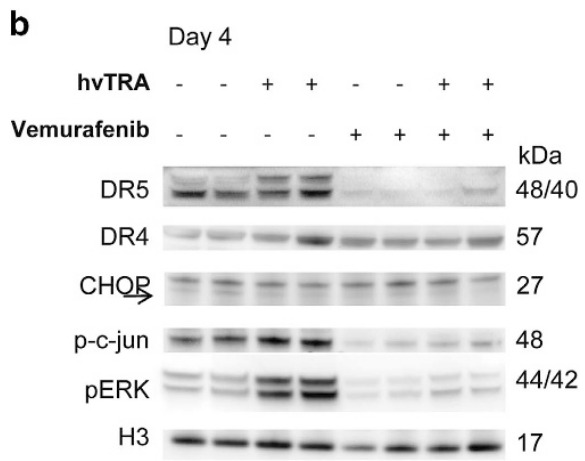

Figure 4. Evaluation of apoptotic proteins in response to hvTRA, vemurafenib or the combination measured by western immunoblot. Western immunoblot detection of DR5, DR4, CHOP, p-c-jun and pERK to investigate the Melmet 5 xenografts response to hvTRA and vemurafenib after (a) 1 day or (b) 4 days of treatment with vemurafenib $(50 \mathrm{mg} / \mathrm{kg}$ twice a day), hvTRA $(3 \mathrm{mg} / \mathrm{kg})$ or the combination. CHOP is indicated by an arrow. $\mathrm{H} 3$ was used as loading control. Tumors were harvested $2 \mathrm{~h}$ after the last treatment. Each lane in a and $\mathbf{b}$ represents one individual tumor. 


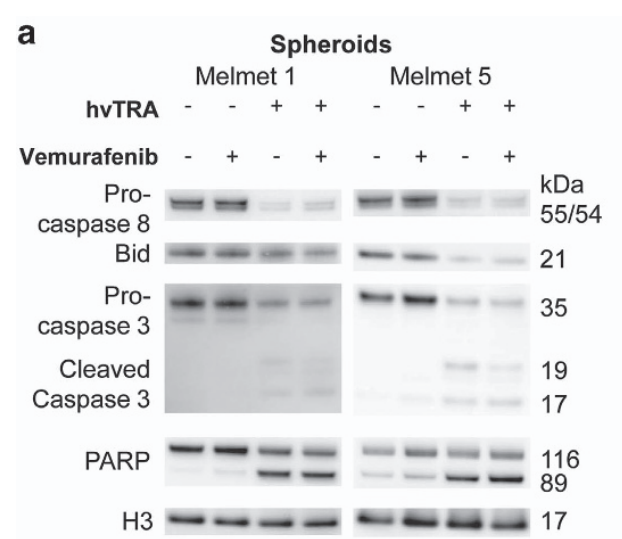

C
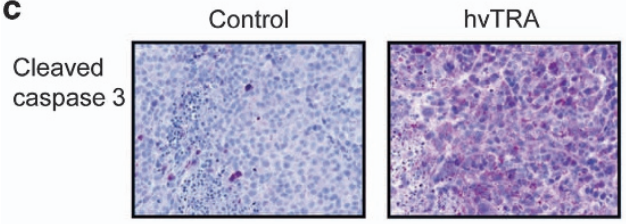

d

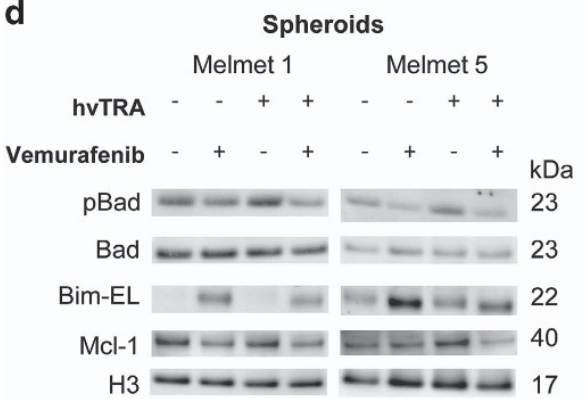

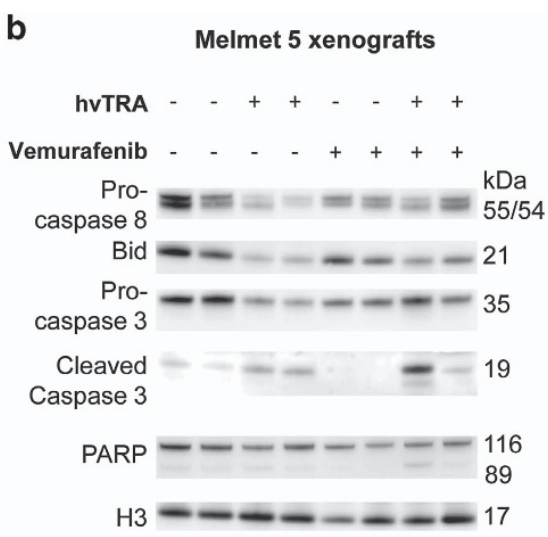

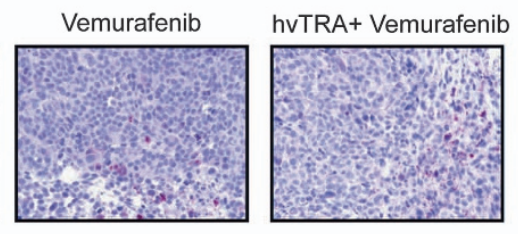

e

Melmet 5 xenografts

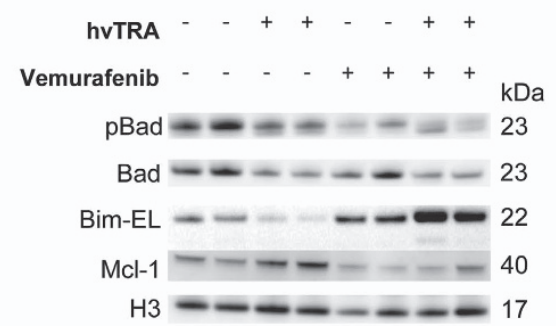

Figure 5. Evaluation of proteins in the extrinsic and intrinsic apoptotic pathways in response to hvTRA and vemurafenib treatment. Western immunoblot of proteins in the (a) extrinsic and (d) intrinsic apoptotic pathways in Melmet 1 and Melmet 5 spheroids in response to hvTRA $(0.5 \mu \mathrm{g} / \mathrm{ml})$, vemurafenib $(0.3 \mu \mathrm{M})$ or the combination after $24 \mathrm{~h}$ of treatment. Two biological parallels of the spheroid experiments were performed. The results presented in (a and $\mathbf{d}$ ) are from the same cell lysate. H3 was used as loading control. (b) Western immunoblot of proteins in the extrinsic and (e) intrinsic apoptotic pathway in Melmet 5 xenografts treated with hvTRA $(3 \mathrm{mg} / \mathrm{kg}), \mathrm{vemurafenib}(50 \mathrm{mg} / \mathrm{kg}$ twice a day) or the combination. Each lane in $\mathbf{b}$ and $\mathbf{e}$ represents one individual tumor. The same cell lysate is used in $\mathbf{b}$ and $\mathbf{e}$. $\mathrm{H} 3 \mathbf{w a s}$ used as loading control. (c) Immunohistochemical detection of cleaved caspase 3 in Melmet 5 xenografts treated with hvTRA (3 mg/kg), vemurafenib $(50 \mathrm{mg} / \mathrm{kg}$ twice a day) or the combination. Tumors were harvested on days $4,2 \mathrm{~h}$ after the last treatment.

in vivo, ${ }^{20}$ but has not been studied in malignant melanoma previously. In the present study, we have investigated the efficacy of hvTRA in melanoma models of different complexity. We confirmed hvTRA-induced growth inhibition and apoptosis induction in vitro, and demonstrated reduced lung tissue colonization in the ex vivo pulmonary metastasis assay. The cell line Melmet 5 showed a modest response to hvTRA in vitro, but when grown as xenografts, hvTRA showed a strong and sustained growth inhibitory effect. The Melmet 5 xenografts regressed in size in all four in vivo experiments performed, demonstrating consistent efficacy and reproducible effect of hvTRA. An increase in DR5 protein level was observed in response to hvTRA treatment and may contribute to strengthen the already existing initial growth inhibitory effect. Furthermore, hvTRA showed efficacy in a substantial fraction of melanoma biopsies grown as spheroids ex vivo, implying potency of hvTRA, at least in subgroups of melanoma patients.

Different strategies have been applied to increase the efficacy of TRAs. Increasing DR5 expression ${ }^{39,40}$ or manipulating the apoptosis mechanisms ${ }^{30,41}$ leading to activation of both the extrinsic and intrinsic apoptotic pathways are two strategies. In vitro studies evaluating the combination of MAPK/ERK inhibitors and TRAs have been published with conflicting conclusions. Berger et al. ${ }^{21}$ show that BRAF inhibition can overcome resistance to TRAIL-induced apoptosis, while Oh et al. ${ }^{32}$ demonstrated that BRAF inhibition downregulates DR5 in vitro, and thereby inhibited TRAs. Our data indicate that hvTRA induces apoptosis through the extrinsic apoptotic pathway, but inhibit apoptosis activation through the intrinsic pathway by increasing the anti-apoptotic and reducing the pro-apoptotic proteins involved in the regulation of this pathway. However, when hvTRA is combined with vemurafenib the effect on the intrinsic regulating proteins was counteracted and thereby, the cells should be more primed for apoptosis induction. A strong, initial reduction in tumor volume show that the combination is potent, but the effect is not sustained, which can be related to a vemurafenib-induced reduction in DR5 expression. The limited effect of hvTRA in combination with vemurafenib is also demonstrated by reduced cleavage of pro-caspases 3 and 8 and Bid. c-jun and CHOP are transcription factors involved in regulation of $\mathrm{DR} 5,{ }^{37}$ and regulated by the MAPK/ERK pathway. An increase in $\mathrm{p}$-c-jun was observed in response to hvTRA, while a decrease was observed in all samples treated with vemurafenib, corresponding well with the observed increase and decrease in DR5 levels. We also observed a 
a

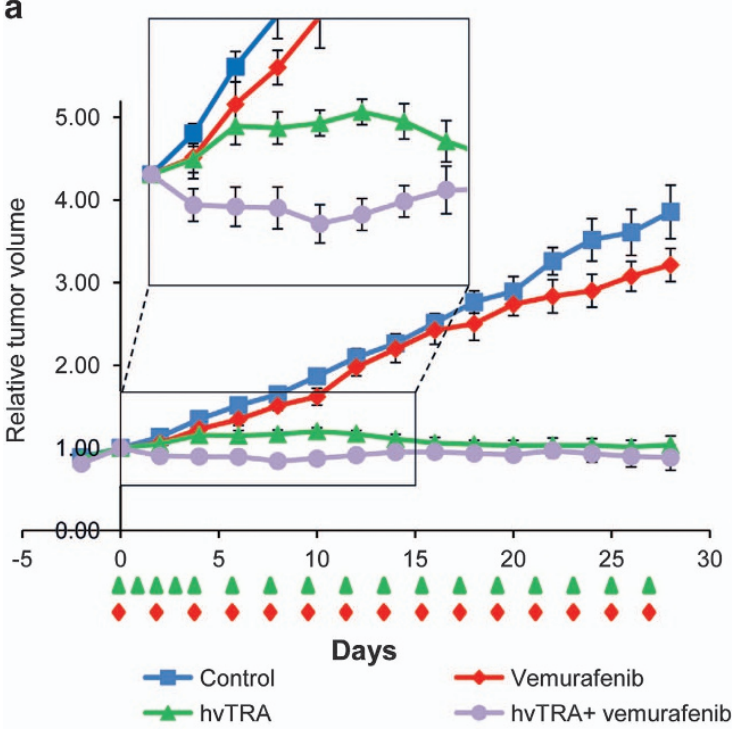

C

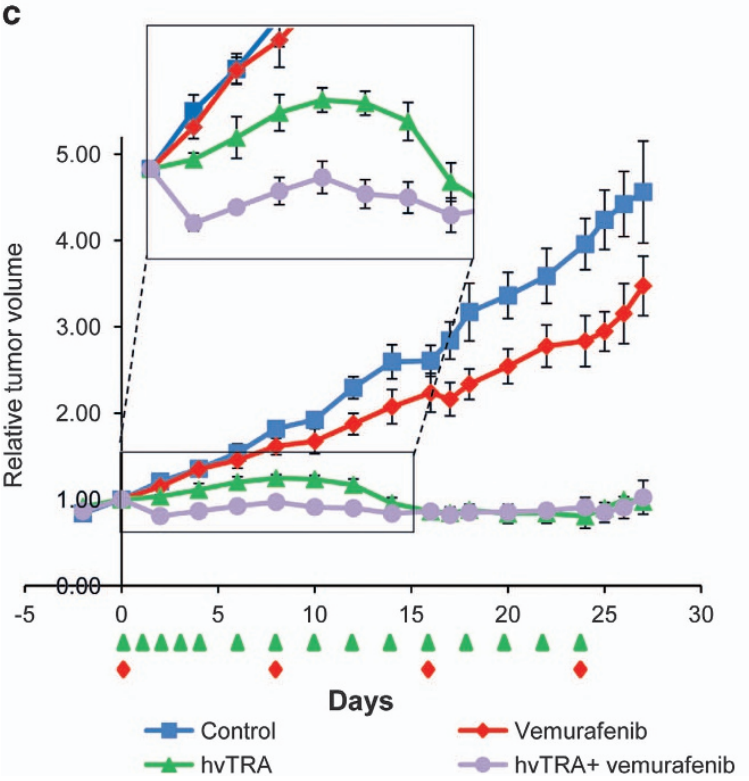

b
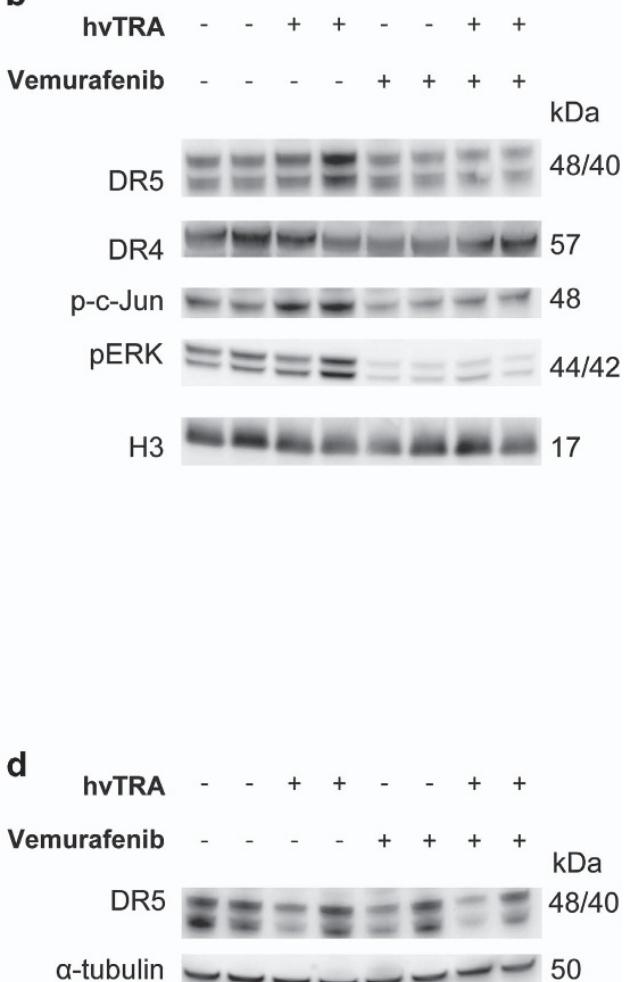

Figure 6. Growth reducing capacity of hvTRA, vemurafenib and the combination with reduced concentrations of vemurafenib. Melmet 5 xenografts were treated with hvTRA ( ) $(3 \mathrm{mg} / \mathrm{kg})$, vemurafenib ( $\downarrow)((\mathbf{a}) 50 \mathrm{mg} / \mathrm{kg}$ every second day) or (c) $50 \mathrm{mg} / \mathrm{kg}$ twice a day every 8 th days) or the combination of hvTRA and vemurafenib $(\bullet)$. The treatment schedule is indicated in the figure by $\Delta$ and $\downarrow$. The tumors were measured twice or thrice a week using a caliper, and are presented as relative tumor volume related to the tumor volume at the initiation of the treatment. Eight tumors are included in each group in a, and five tumors in each group in c. Error bars represent \pm S.E.M. (b) Western immunoblot detection of DR5, DR4, p-c-jun and pERK in response to hvTRA and vemurafenib treatment (50 mg/kg every second day). Tumors were harvested on day 4, $2 \mathrm{~h}$ after the last treatment. H3 was used as loading control. (d) Western immunoblot detection of DR5 in response to hvTRA and vemurafenib treatment $(50 \mathrm{mg} / \mathrm{kg}$ twice a day every 8 th days). Tumors were harvested 8 days after the last treatment. $\alpha$-tubulin was used as loading control. Each lane in $\mathbf{b}$ and $\mathbf{d}$ represents one individual tumor.

downregulation of DR5 in vitro, but at vemurafenib concentrations higher than what was applied in the combination experiments.

Based on our observation and previously published data showing transient reduction in DR5 expression after MAPK/ERK inhibition, ${ }^{32}$ additional in vivo experiments with altered administration of vemurafenib were performed. Although DR5 expression was less effected with reduced vemurafenib dosing, a prolonged cytotoxic effect of the combination was not observed. The combination did induce an initial reduction in tumor volume exceeding the effect of hvTRA alone, but it was not sustained and addition of vemurafenib did not increase the growth-inhibiting effects of hvTRA in long-term experiments, suggesting that continuous dosing of vemurafenib and TRAs is not a desirable combination.

Vemurafenib usually has a very good initial effect in mutated BRAF patients, but the majority develops resistance within months. There is therefore a need to identify means to avoid or delay acquired resistance, and also alternative treatment options for patients having developed resistance and not eligible to receive immune therapy. Our results open for further investigation of TRA given on first day of vemurafenib treatment. This might, as demonstrated in the present work, enhance the growth-inhibiting effect, which could alter the surviving tumor cell population and possibly affect the progression-free survival of the patients. 


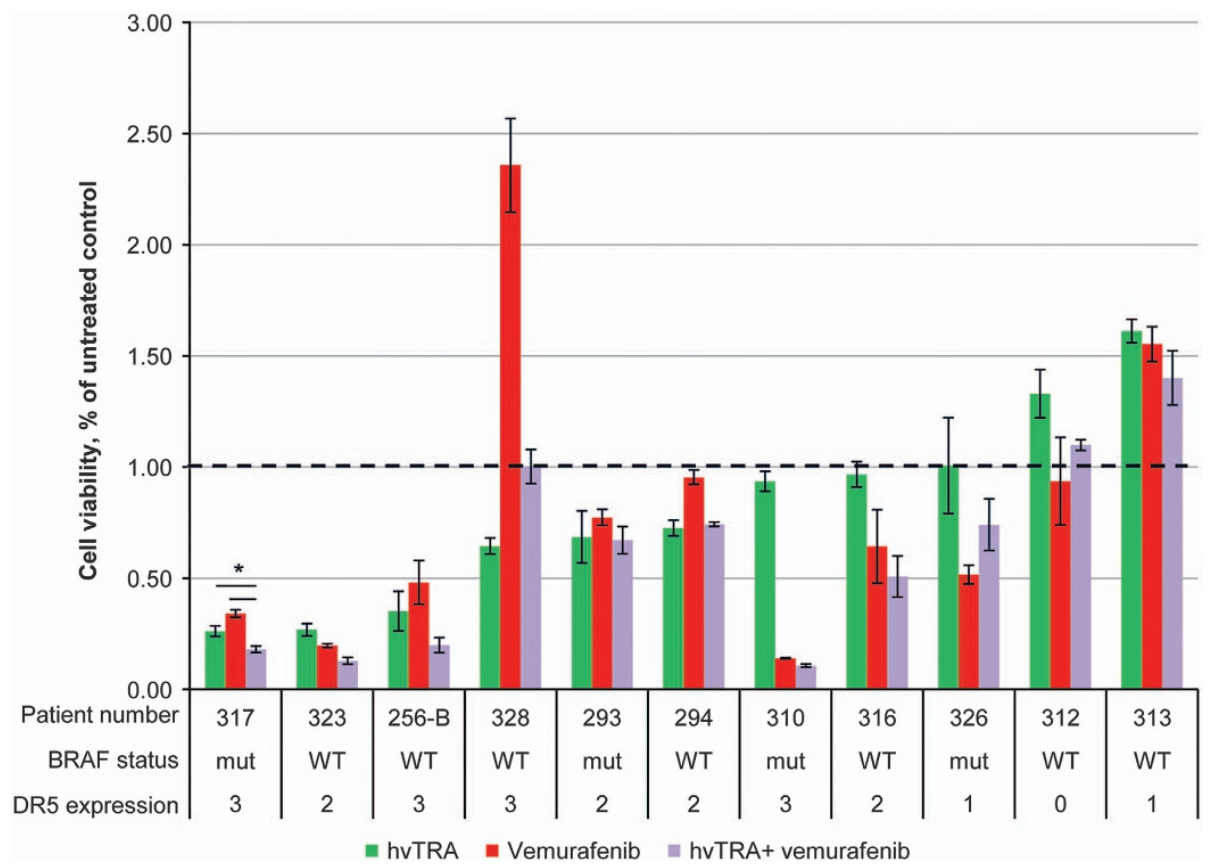

Figure 7. Sensitivity to hvTRA, vemurafenib and the combination in patient biopsies. Eleven biopsies from lymph node metastases from melanoma patients were made into single-cell suspensions, and seeded in 96 -well round-bottom plates and exposed to hvTRA ( $5 \mu \mathrm{g} / \mathrm{ml}$ ), vemurafenib $(5 \mu \mathrm{M})$ or the combination. Cell viability was measured after $120 \mathrm{~h}$ using CellTiter-Glo Luminescent assay. Three technical replicates were performed, and the error bars represent \pm S.D. The luminescent signal from untreated control cells from each patient sample were set to 1, indicated by the dotted line. For DR5 expression 0; not expressed, $1 ; 0-10 \%$ of the cells express DR5, $2 ; 10-50 \%$ of the cells express DR5, 3; $>50 \%$ of the cells express DR5. Patient biopsies 294 and 328 have mutated NRAS. ${ }^{*} P<0.05$.

Furthermore, hvTRA should be considered as a mono treatment for subgroups of melanoma patients. It has previously been shown that cells with mutated BRAF express higher DR5 levels, and respond better to DR5 targeting therapy than cells with wild-type BRAF. ${ }^{37}$ This implies that TRAIL receptor agonists could be a valuable option for patients with mutated BRAF, and should be evaluated in patients with acquired resistance against vemurafenib as they may presumably have high DR5 levels. In our ex vivo experiments utilizing patient tissue, DR5 expression was necessary, but did not guarantee response to hvTRA, implying that additional predictive biomarkers would be valuable to identify responders. We did not observe an association between mutated BRAF and DR5 levels, but the number of patients was low and a more thorough investigation on the correlation between mutation status and receptor expression is needed before any conclusion can be made. Furthermore, our patients were only tested for the most common mutations of BRAF, V600E/K, and not the more rare mutations $\mathrm{V} 600 \mathrm{D} / \mathrm{R}$ also responsive to vemurafenib. ${ }^{42}$ Thus, it is possible that some of the samples annotated as wild type may have mutations explaining the response to vemurafenib.

Despite the new drugs approved for metastatic melanoma in recent years, new treatment modalities are needed as the available options only show efficacy in a subgroup of patients and development of resistance is a significant problem. The data presented herein show that hvTRA can induce cell death in vitro and efficiently inhibit tumor growth in vivo. Synergistic or additive effects were observed when hvTRA was combined with vemurafenib in vitro, while reduced DR5 expression hampered the effect in vivo. This is valuable new information on the efficacy of hvTRA in melanoma and, to our knowledge, the first study where a TRA has been combined with a MAPK/ERK inhibitor in vivo. Additional studies are warranted to conclude if it is possible to take advantage of the cytotoxic effect of combining hvTRA and vemurafenib before vemurafenib-mediated downregulation of
DR5. Furthermore, hvTRA as mono therapy should be evaluated more thoroughly to identify predictive biomarkers for selecting subgroups of patients for whom hvTRA will show efficacy.

\section{MATERIALS AND METHODS}

Cell lines and culture conditions

The malignant melanoma cell lines Melmet 5 and LOX were established from lymph node metastases, while Melmet 1 was established from a subcutaneous metastasis, surgically removed at the Norwegian Radium Hospital (Oslo University Hospital, Norway) as described previously. ${ }^{43,44}$ The cell lines Patient-3-pre and Patient-3-post were a kind gift from Professor Peter Hersey (Royal North Shore Hospital, Sydney, Australia), and established as described previously. ${ }^{45}$ WM1366 was kindly provided by Professor Meenhard Herlyn (the Wistar institute, Philadelphia, PA, USA), whereas the $A 375$ cell line was obtained from the American Type Culture Collection (Rockville, MD, USA). Green fluorescent protein (GFP) and luciferase (Luc) expressing Melmet 5 cells were generated by transducing the cells with lentivirus carrying a human ferritin promoter-driven GFP-Luc construct (described previously ${ }^{46}$ ) and kindly provided by Dr. Glen Merlino $(\mathrm{NIH}, \mathrm{MD}, \mathrm{USA})$. The cells were grown in RPMI- 1640 medium (SigmaAldrich, St. Louis, MO, USA), supplemented with $10 \%$ fetal bovine serum (Sigma-Aldrich) and 1\% GlutaMax (Life Technologies, Carlsbad, CA, USA) at $37^{\circ} \mathrm{C}$ with $5 \% \mathrm{CO}_{2}$.

\section{Chemicals and drugs}

The mutated RAF inhibitor vemurafenib (S1267) was purchased from Selleck (Selleck Chemicals, Houston, TX, USA), while the TRA hvTRA was a kind gift from Dr. Oliver Hill, Apogenix, Heidelberg, Germany. The hvTRA Fc-fusion protein was produced as described recently. ${ }^{20}$ For in vitro experiments vemurafenib was dissolved in DMSO, and then further diluted with RMPI-1640 medium. For in vivo experiments vemurafenib was dissolved in DMSO to a concentration of $50 \mathrm{mg} / \mathrm{ml}$, and then further diluted in $0.5 \%$ methylcellulose. hvTRA was diluted in PBS for in vivo experiments and in RPMI-1640 for in vitro experiments. 
Cell viability

Cell viability after 2D culturing was measured using CellTiter 96 Aqueous One Solution (MTS; Promega, Madison, WI, USA). The cells were seeded in 96-well plates (Thermo Fisher Scientific, Rockford, IL, USA). The next day vemurafenib and hvTRA at concentrations indicated in the figure legends were added. The cells were incubated for $72 \mathrm{~h}$ before addition of CellTiter 96 Aqueous One Solution. After $\sim 2 \mathrm{~h}$ incubation at $37^{\circ} \mathrm{C}$ the absorbance was measured at $490 \mathrm{~nm}$ using a microplate reader (Victor ${ }^{2} 1420$ Multilabel Counter, Perkin Elmer, Waltham, MA, USA).

Cell viability after 3D culturing was measured using CellTiter-Glo Luminescent Cell Viability Assay (Promega). Briefly, the cells were cultured in ultra-low attachment round-bottom 96-well plates (3000 cells/well; Sigma-Aldrich); Melmet 1 was seeded in RPMI-1640 medium containing $2 \%$ Matrigel (BD Bioscience, San Jose, CA, USA), while Melmet 5 were cultured without Matrigel. After 4 days of cultivation, hvTRA and/or vemurafenib were added at concentrations indicated in the figure legends. Following $96 \mathrm{~h}$ treatment viability was assessed using CellTiter-Glo Luminescent Cell Viability Assay as described by the manufacturer. Luminescence was measured using a microplate reader (Victor ${ }^{2} 1420$ Multilabel Counter, Perkin Elmer). Cell viability is reported as percentage viable cells in treated as compared to untreated control samples. Both $2 \mathrm{D}$ and $3 \mathrm{D}$ experiments were performed in quadruplicates, and repeated at least three times.

\section{Calcusyn analysis}

The Chou and Talalay $\mathrm{Cl}$ was used to determine possible synergism. Synergistic effect is defined as $\mathrm{Cl}$ values $<1$, antagonistic effect as $\mathrm{Cl}$ values $>1$ and additive effect as $\mathrm{Cl}=1 .{ }^{47}$ The $\mathrm{Cl}$ values are calculated using Calcusyn software (BioSoft, Ferguson, MO, USA).

\section{Pulmonary metastasis assay}

The pulmonary metastasis assay (PUMA) was performed as described earlier. ${ }^{33}$ GFP-Luc labeled Melmet 5 cells $\left(5 \times 10^{5}\right)$ were injected intravenously (i.v). Fifteen minutes thereafter the mice were placed under sevofluran anesthesia (5\%) and given a lethal injection of pentobarbital intraperitoneally. Trachea and lungs were exposed, and a $24 \mathrm{G}$ Neoflon was inserted in the trachea under sterile conditions. The cell culture media M199 2X (Sigma-Aldrich), supplemented with $2 \mu \mathrm{g} / \mathrm{ml}$ crystalline bovine insulin, $0.2 \mu \mathrm{g} / \mathrm{ml}$ hydrocortison, $1 \mu \mathrm{g} / \mathrm{ml}$ retinyl acetat and $200 \mathrm{U} / \mathrm{ml}$ penicillin/streptomycin was mixed $1: 1$ with low melting agarose $(1.2 \%$; Thermo Fisher Scientific), and slowly injected into the lungs. When lungs were completely dilated, the trachea was closed off with a 5.0 suture (Polysorb, Covidien, Dublin, Ireland). The lungs were cut out and placed in cold PBS with $100 \mathrm{U} / \mathrm{ml}$ penicillin/streptomycin. After $20 \mathrm{~min}$, they were divided into $1 \mathrm{~mm}$-thick slices using a Manual digital tissue chopper (Leica Biosystems, Nussloch, Germany), and placed on a gel foam (Pfizer, New York, NY, USA), which had been soaked in culture media M199 (1 ; Thermo Fisher Scientific), supplemented with $1 \mu \mathrm{g} / \mathrm{ml}$ crystalline bovine insulin, $0.1 \mu \mathrm{g} / \mathrm{ml}$ hydrocortison, $0.5 \mu \mathrm{g} / \mathrm{ml}$ retinyl acetat and $100 \mathrm{U} / \mathrm{ml}$ penicillin/streptomycin, for $2 \mathrm{~h}$. The slices were then exposed to either just media (control) or $0.25 \mu \mathrm{g} / \mathrm{ml} \mathrm{hvTRA}$. The slices were cultured at $37{ }^{\circ} \mathrm{C}, 5 \%$ $\mathrm{CO}_{2}$ for 2 weeks. The medium was changed every second day, and the lung slices were then turned over. The fluorescent microscope Olympus IX81 (Olympus, Tokyo, Japan) was used for imaging, and ImageJ (National Institute of Health, MD, USA) was used to quantify the intensity of the colonies. The settings on the microscope were changed each day pictures were taken and the intensity of treated samples can therefore only be compared with pictures of controls taken at the same day. A threshold value was set, and only cells with intensity over the threshold were chosen by the program ImageJ for further calculation. Intensity is reported as intensity in treated slices relative to untreated slices. The experiments were repeated four times, each with four technical replicates.

\section{In vivo studies}

All procedures and experiments involving animals were approved by the National Animal Research Authority, and conducted according to the regulations of European Laboratory Animals Science Association. Female athymic nude foxn1 nu mice were bred at the local Department of Comparative Medicine, OUS, and kept in a specific pathogen-free environment. Food and water were supplied ad libitum.

In all, $2.5 \times 10^{6}$ Melmet 5 cells were injected subcutaneously on the flank. When the tumors reached $\sim 85 \mathrm{~mm}^{3}$, the mice were divided into three or four groups. If mice had two tumors the mean volume was used for statistical analysis. hvTRA $(0.3$ or $3 \mathrm{mg} / \mathrm{kg}$ ) was given i.v., while vemurafenib was given orally $(50 \mathrm{mg} / \mathrm{kg}$ ). For treatment regimen see Figures 3 and 6 . Controls were given $10 \%$ DMSO in $0.5 \%$ methylcellulose orally and PBS i.v. for as long as the other mice received treatment. Tumor volume was measured twice or three times a week, and calculated using the formula: width ${ }^{2} \times$ length $\times 0.5$. Two tumors from each group were harvested on day 1,4 or $10,2 \mathrm{~h}$ after the last treatment. Half of each tumor was snap-frozen in $\mathrm{N}_{2}$ for western immunoblot analysis while the other half was fixed in $10 \%$ formalin for immunohistochemical analyses. At the end of the experiments the mice were euthanized by dislocation of the neck.

\section{Western immunoblot analysis}

Fresh frozen tumors from mice were crushed to powder using mortar and pistil. Both tumors and cells harvested from in vitro experiments were lysed in buffer $(20 \mathrm{mM}$ Tris- $\mathrm{HCl}$ pH 7.5, $137 \mathrm{mM} \mathrm{NaCl}, 100 \mathrm{mM} \mathrm{NaF}, 10 \%$ glycerol and $1 \%$ NP-40 supplemented with cOmplete Mini and PhosSTOP (both from Roche, Basel, Switzerland) for $1 \mathrm{~h}$ on ice with vortexing every $15 \mathrm{~min}$ before sonication. Samples were centrifuged and the supernatant transferred to new tubes and stored at $-70^{\circ} \mathrm{C}$. $10-50 \mu \mathrm{g}$ protein were separated using NuPAGE novex Bis-Tris 4-12\% gel (Life Technologies) and thereafter transferred to a nitrocellulose membrane using the iBlot Dry blotting system (Thermo Fisher Scientific). Membranes were blocked in either $5 \%$ dry milk or $5 \%$ BSA in TBST $(0.5 \%$ Tween 20$)$ for $1 \mathrm{~h}$ and incubated overnight at $4{ }^{\circ} \mathrm{C}$ with primary antibodies. Antibodies used were: DR5 (\#3696), pERK (\#9102), pBad ${ }^{\text {ser112 }}$ (\#4366), Bad (\#9292), Bid (\#2002), Bim (\#2933), caspase 3 (\#9664), cl. caspase 3 (\#9662), PARP (\#9542), Mcl-1 (\#4572), CHOP (\#5554), p-c-jun (\#3270), Histone 3 (H3) (\#4499), (all from Cell Signaling Technology, Danvers, MA, USA), caspase 8 (ALX-804-242; Enzo Life Sciences, Farmingdale, NY, USA) $a$-tubulin (\#CP06; Merck Millipore, Billerica, MA, USA) and DR4 (ab\#8414; Abcam, Cambridge, UK). Following primary hybridization, membranes were washed $3 \times 10 \mathrm{~min}$ in TBST before applying appropriate HRP-conjugated secondary antibody for $1 \mathrm{~h}$ at room temperature. Membranes were then washed for $3 \times 10 \mathrm{~min}$. The signals were developed with SuperSignal West Dura Extended Duration Substrate (Thermo Fisher Scientific) and visualized in G:BOX (Syngene, Cambridge, UK).

\section{Immunohistochemistry}

Formalin-fixed tumors were dehydrated and embedded in paraffin. Subsequently, 3- $\mu \mathrm{m}$ tumor sections were prepared and placed on a microscope slide. Prior to incubation with primary antibody, paraffin sections were dewaxed in xylene and rehydrated in ethanol/water. For antigen retrieval, the tumor sections were treated at $99^{\circ} \mathrm{C}$ for $25 \mathrm{~min}$ in citrate buffer (Target Retrieval Solution, pH 6.0, DAKO, Glostrup, Denmark). The following primary antibody was used: rabbit anti-cleaved caspase 3 (BD Biosciences). The tumor sections were incubated with the primary rabbit antibody diluted $1: 100$ in blocking buffer (PBS+20 mg/ml BSA $+1 \mathrm{mg} / \mathrm{ml}$ human $\mathrm{lgG}$ ) for $60 \mathrm{~min}$ at room temperature. After a PBS washing step, specific binding of the primary antibody was visualized using an anti-rabbit biotinylated secondary antibody (Southern Biotech, Birmingham, AL, USA) and streptavidin alkaline phosphatase (BioGenex, Fremont, CA, USA). The FAST-Red substrate system (DAKO) was used as the substrate for the alkaline phosphatase, which produced a red precipitate at antibody-binding sites. Sections were then counterstained with Mayer's hematoxylin and mounted with glycerin-gelatin. A rabbit isotype control antibody was used for control staining.

\section{Ex vivo patient samples}

All patients included in the study got relevant information and have signed a written consent. The study was approved by the Regional Ethical Committee (approval no: 2012/2309). Freshly operated tumor tissue from lymph node metastases was mechanically disaggregated and treated with collagenase $(700 \mathrm{U} / \mathrm{ml}$; Worthington Biochemical Corporation, Lakewood, $\mathrm{NJ}$, USA) overnight at $37^{\circ} \mathrm{C}$ in $5 \% \mathrm{CO}_{2}$. The cell suspension was filtered through a $100 \mu \mathrm{m}$ nylon cell strainer (BD-Falcon, Franklin Lakes, NJ, USA) to remove debris and large cell clumps. If required, red blood cells were removed by treating the cell suspension with ACK lysis buffer according to the manufacturer's instruction (Lonza, Walkersville, MD, USA). The cells were washed in cold PBS and resuspended in RPMI-1640 medium supplemented with $5 \%$ FCS, $2 \mathrm{mM}$ L-glutamine, and $50 \mathrm{U} / \mathrm{ml}$ each of penicillin/streptavidin (the latters from Lonza, Ververiers, Belgium). Approximately 20000 live cells, assessed by trypan blue exclusion, was 
seeded in round-bottom 96-well ultra-low adhesion plates in the presence/ absence of hvTRA and vemurafenib alone and in combination. Effect on viability was examined using the CellTiter-Glo Luminescent Cell Viability Assay as previously described after 5 days. RT-PCR were used to decide the BRAF mutation status of the patient material. The test only detects the mutations V600E and V600K, and does not differentiate between these two mutations.

\section{Statistical analysis}

One-Way ANOVA Tukey HSD tests were used for statistical analyses, which were performed using IBM SPSS Statistics 21 (IBM, Anmork, NY, USA). Differences were considered statistically significant if $P$-values were below 0.05 , and are indicated by $*$ in the figures.

\section{ACKNOWLEDGEMENTS}

This work was supported by grants from the Norwegian Cancer Society (Grant No (42185263650)) and the Research Council of Norway (Project No (222262/F20). We thank Professor Peter Hersey (Royal North Shore Hospital, University of Sydney, Australia) for providing Patient-3-pre and Patient-3-post melanoma cell lines, Elisabeth Emilsen and Anna Rosness for technical assistance and Michael Kluge for valuable advice.

\section{AUTHOR CONTRIBUTIONS}

KGF contributed to design, acquiring and interpretation of data as well as drafting of the manuscript. VAF contributed to acquiring and interpretation of data and revision of manuscript. LP contributed to interpretation of data and revision of manuscript. $\mathrm{OH}$ contributed to revision of manuscript and supplied hvTRA. JS contributed to acquiring of data. GMM contributed to design, interpretation of data and revision of the manuscript. BE contributed to design, acquiring and interpretation of data and revision of the manuscript. All authors read and approved the final version of the manuscript. In Figures $1 \mathrm{a}, 2 \mathrm{a}$ and $4 \mathrm{~b}$ $B E$ generated the data. In Figure $5 c$, JS generated the data. In Figure 7, VAF generated the data. KGF generated the data in Figures 1-6 and assembled the panels.

\section{COMPETING INTERESTS}

O Hill and J Sykora are employees at Apogenix, which has developed hvTRA. The remaining authors declare no conflict of interest.

\section{REFERENCES}

1 Chapman PB, Hauschild A, Robert C, Haanen JB, Ascierto P, Larkin J et al. Improved survival with vemurafenib in melanoma with BRAF V600E mutation. N Engl J Med 2011; 364: 2507-2516.

2 Hodi FS, O'Day SJ, McDermott DF, Weber RW, Sosman JA, Haanen JB et al. Improved survival with ipilimumab in patients with metastatic melanoma. $N$ Engl J Med 2010; 363: 711-723.

3 Walczak H, Miller RE, Ariail K, Gliniak B, Griffith TS, Kubin M et al. Tumoricidal activity of tumor necrosis factor-related apoptosis-inducing ligand in vivo. Nat Med 1999; 5: 157-163.

4 Ashkenazi A, Pai RC, Fong S, Leung S, Lawrence DA, Marsters SA et al. Safety and antitumor activity of recombinant soluble Apo2 ligand. J Clin Invest 1999; 104: 155-162.

5 Griffith TS, Stokes B, Kucaba TA, Earel JK Jr., VanOosten RL, Brincks EL et al. TRAIL gene therapy: from preclinical development to clinical application. Curr Gene Ther 2009; 9: 9-19.

6 Subbiah V, Brown RE, Buryanek J, Trent J, Ashkenazi A, Herbst R et al. Targeting the apoptotic pathway in chondrosarcoma using recombinant human Apo2L/ TRAIL (dulanermin), a dual proapoptotic receptor (DR4/DR5) agonist. Mol Cancer Ther 2012; 11: 2541-2546.

7 Soria JC, Mark Z, Zatloukal P, Szima B, Albert I, Juhasz E et al. Randomized phase II study of dulanermin in combination with paclitaxel, carboplatin, and bevacizumab in advanced non-small-cell lung cancer. J Clin Oncol 2011; 29: 4442-4451.

8 Trarbach T, Moehler M, Heinemann V, Kohne $\mathrm{CH}$, Przyborek M, Schulz C et al. Phase II trial of mapatumumab, a fully human agonistic monoclonal antibody that targets and activates the tumour necrosis factor apoptosis-inducing ligand receptor-1 (TRAIL-R1), in patients with refractory colorectal cancer. Br J Cancer 2010; 102: 506-512.
9 Forero-Torres A, Shah J, Wood T, Posey J, Carlisle R, Copigneaux C et al. Phase I trial of weekly tigatuzumab, an agonistic humanized monoclonal antibody targeting death receptor 5 (DR5). Cancer Biother Radiopharm 2010; 25: 13-19.

10 Plummer R, Attard G, Pacey S, Li L, Razak A, Perrett R et al. Phase 1 and pharmacokinetic study of lexatumumab in patients with advanced cancers. Clin Cancer Res 2007; 13: 6187-6194.

11 Herbst RS, Eckhardt SG, Kurzrock R, Ebbinghaus S, O'Dwyer PJ, Gordon MS et al. Phase I dose-escalation study of recombinant human Apo2L/TRAIL, a dual proapoptotic receptor agonist, in patients with advanced cancer. J Clin Oncol 2010; 28: 2839-2846.

12 Merchant MS, Geller Jl, Baird K, Chou AJ, Galli S, Charles A et al. Phase I trial and pharmacokinetic study of lexatumumab in pediatric patients with solid tumors. J Clin Oncol 2012; 30: 4141-4147.

13 von Pawel J, Harvey JH, Spigel DR, Dediu M, Reck M, Cebotaru CL et al. Phase II trial of mapatumumab, a fully human agonist monoclonal antibody to tumor necrosis factor-related apoptosis-inducing ligand receptor 1 (TRAIL-R1), in combination with paclitaxel and carboplatin in patients with advanced non-small-cell lung cancer. Clin Lung Cancer 2014; 15: 188-196 e182.

14 Wilson NS, Yang B, Yang A, Loeser S, Marsters S, Lawrence D et al. An Fcgamma receptor-dependent mechanism drives antibody-mediated target-receptor signaling in cancer cells. Cancer Cell 2011; 19: 101-113.

15 Kurbanov BM, Fecker LF, Geilen CC, Sterry W, Eberle J. Resistance of melanoma cells to TRAIL does not result from upregulation of antiapoptotic proteins by NF-kappaB but is related to downregulation of initiator caspases and DR4. Oncogene 2007; 26: 3364-3377.

16 Kurbanov BM, Geilen CC, Fecker LF, Orfanos CE, Eberle J. Efficient TRAIL-R1/DR4mediated apoptosis in melanoma cells by tumor necrosis factor-related apoptosis-inducing ligand (TRAIL). J Invest Dermatol 2005; 125: 1010-1019.

17 Hornle M, Peters N, Thayaparasingham B, Vorsmann H, Kashkar H, Kulms D. Caspase-3 cleaves XIAP in a positive feedback loop to sensitize melanoma cells to TRAIL-induced apoptosis. Oncogene 2011; 30: 575-587.

18 Zhang XD, Zhang XY, Gray CP, Nguyen T, Hersey P. Tumor necrosis factor-related apoptosis-inducing ligand-induced apoptosis of human melanoma is regulated by smac/DIABLO release from mitochondria. Cancer Res 2001; 61: 7339-7348.

19 Song JJ, An JY, Kwon YT, Lee YJ. Evidence for two modes of development of acquired tumor necrosis factor-related apoptosis-inducing ligand resistance. Involvement of BCl-xL. J Biol Chem 2007; 282: 319-328.

20 Gieffers C, Kluge M, Merz C, Sykora J, Thiemann M, Schaal R et al. APG350 induces superior clustering of TRAIL receptors and shows therapeutic antitumor efficacy independent of cross-linking via Fcgamma receptors. Mol Cancer Ther 2013; 12: 2735-2747.

21 Berger A, Quast SA, Plotz M, Kuhn NF, Trefzer U, Eberle J. RAF inhibition overcomes resistance to TRAIL-induced apoptosis in melanoma cells. J Invest Dermatol 2014; 134: 430-440.

22 Engesaeter B, Engebraaten O, Florenes VA, Maelandsmo GM. Dacarbazine and the agonistic TRAIL receptor-2 antibody lexatumumab induce synergistic anticancer effects in melanoma. PLoS One 2012; 7: e45492.

23 Gillespie S, Borrow J, Zhang XD, Hersey P. Bim plays a crucial role in synergistic induction of apoptosis by the histone deacetylase inhibitor SBHA and TRAIL in melanoma cells. Apoptosis 2006; 11: 2251-2265.

24 Tochigi M, Inoue T, Suzuki-Karasaki M, Ochiai T, Ra C, Suzuki-Karasaki Y. Hydrogen peroxide induces cell death in human TRAIL-resistant melanoma through intracellular superoxide generation. Int J Oncol 2013; 42: 863-872.

25 Graves JD, Kordich JJ, Huang TH, Piasecki J, Bush TL, Sullivan T et al. Apo2L/TRAIL and the death receptor 5 agonist antibody AMG 655 cooperate to promote receptor clustering and antitumor activity. Cancer Cell 2014; 26: 177-189.

26 Boisvert-Adamo K, Aplin AE. Mutant B-RAF mediates resistance to anoikis via Bad and Bim. Oncogene 2008; 27: 3301-3312.

27 Shao Y, Aplin AE. Akt3-mediated resistance to apoptosis in B-RAF-targeted melanoma cells. Cancer Res 2010; 70: 6670-6681.

28 VanBrocklin MW, Verhaegen M, Soengas MS, Holmen SL. Mitogen-activated protein kinase inhibition induces translocation of Bmf to promote apoptosis in melanoma. Cancer Res 2009; 69: 1985-1994.

29 Cartlidge RA, Thomas GR, Cagnol S, Jong KA, Molton SA, Finch AJ et al. Oncogenic BRAF(V600E) inhibits BIM expression to promote melanoma cell survival. Pigment Cell Melanoma Res 2008; 21: 534-544.

30 Wang YF, Jiang CC, Kiejda KA, Gillespie S, Zhang XD, Hersey P. Apoptosis induction in human melanoma cells by inhibition of MEK is caspase-independent and mediated by the Bcl-2 family members PUMA, Bim, and Mcl-1. Clin Cancer Res 2007; 13: 4934-4942.

31 Sheridan C, Brumatti G, Martin SJ. Oncogenic B-RafV600E inhibits apoptosis and promotes ERK-dependent inactivation of Bad and Bim. J Biol Chem 2008; 283: 22128-22135.

32 Oh YT, Deng J, Yue P, Owonikoko TK, Khuri FR, Sun SY. Inhibition of B-Raf/MEK/ ERK signaling suppresses DR5 expression and impairs response of cancer cells to DR5-mediated apoptosis and T cell-induced killing. Oncogene 2015; 35: 459-467. 
33 Mendoza A, Hong SH, Osborne T, Khan MA, Campbell K, Briggs J et al. Modeling metastasis biology and therapy in real time in the mouse lung. J Clin Invest 2010; 120: 2979-2988.

34 Zhang XD, Borrow JM, Zhang XY, Nguyen T, Hersey P. Activation of ERK1/2 protects melanoma cells from TRAIL-induced apoptosis by inhibiting Smac/DIABLO release from mitochondria. Oncogene 2003; 22: 2869-2881.

35 Chitcholtan K, Sykes PH, Evans JJ. The resistance of intracellular mediators to doxorubicin and cisplatin are distinct in $3 \mathrm{D}$ and $2 \mathrm{D}$ endometrial cancer. J Trans/ Med 2012; 10: 38.

36 Mehta G, Hsiao AY, Ingram M, Luker GD, Takayama S. Opportunities and challenges for use of tumor spheroids as models to test drug delivery and efficacy. J Control Release 2012; 164: 192-204.

37 Oh YT, Yue P, Zhou W, Balko JM, Black EP, Owonikoko TK et al. Oncogenic Ras and B-Raf proteins positively regulate death receptor 5 expression through coactivation of ERK and JNK signaling. J Biol Chem 2012; 287: 257-267.

38 Corcoran RB, Rothenberg SM, Hata AN, Faber AC, Piris A, Nazarian RM et al. TORC1 suppression predicts responsiveness to RAF and MEK inhibition in BRAF-mutant melanoma. Sci Transl Med 2013; 5: 196ra198.

39 Jiang CC, Chen LH, Gillespie S, Kiejda KA, Mhaidat N, Wang YF et al. Tunicamycin sensitizes human melanoma cells to tumor necrosis factor-related apoptosisinducing ligand-induced apoptosis by up-regulation of TRAIL-R2 via the unfolded protein response. Cancer Res 2007; 67: 5880-5888.

40 Berger A, Quast SA, Plotz M, Hein M, Kunz M, Langer P et al. Sensitization of melanoma cells for death ligand-induced apoptosis by an indirubin derivativeEnhancement of both extrinsic and intrinsic apoptosis pathways. Biochem Pharmacol 2010; 81: 71-81.

41 Quast SA, Berger A, Buttstadt N, Friebel K, Schonherr R, Eberle J. General sensitization of melanoma cells for TRAIL-induced apoptosis by the potassium channel inhibitor TRAM-34 depends on release of SMAC. PLoS One 2012; 7: e39290.
42 Klein O, Clements A, Menzies AM, O'Toole S, Kefford RF, Long GV. BRAF inhibitor activity in V600R metastatic melanoma. Eur J Cancer 2012; 49: 1073-1079.

43 Prasmickaite L, Skrbo N, Hoifodt HK, Suo Z, Engebraten O, Gullestad HP et al. Human malignant melanoma harbours a large fraction of highly clonogenic cells that do not express markers associated with cancer stem cells. Pigment Cell Melanoma Res 2010; 23: 449-451.

44 Fodstad O, Aamdal S, McMenamin M, Nesland JM, Pihl A. A new experimental metastasis model in athymic nude mice, the human malignant melanoma LOX. Int J Cancer 1988; 41: 442-449.

45 Lai F, Jiang CC, Farrelly ML, Zhang XD, Hersey P. Evidence for upregulation of Bim and the splicing factor SRp55 in melanoma cells from patients treated with selective BRAF inhibitors. Melanoma Res 2012; 22: 244-251.

46 Day CP, Carter J, Bonomi C, Esposito D, Crise B, Ortiz-Conde B et al. Lentivirusmediated bifunctional cell labeling for in vivo melanoma study. Pigment Cell Melanoma Res 2009; 22: 283-295.

47 Chou TC. Theoretical basis, experimental design, and computerized simulation of synergism and antagonism in drug combination studies. Pharmacol Rev 2006; 58 : 621-681.

\section{(c) (i)}

This work is licensed under a Creative Commons Attribution 4.0 International License. The images or other third party material in this article are included in the article's Creative Commons license, unless indicated otherwise in the credit line; if the material is not included under the Creative Commons license, users will need to obtain permission from the license holder to reproduce the material. To view a copy of this license, visit http://creativecommons.org/licenses/ by/4.0/

(C) The Author(s) 2016

Supplementary Information accompanies the paper on the Cell Death and Discovery website (http://www.nature.com/cddiscovery) 